\title{
LEGAL DESIGN THINKING, VISUALES EN LOS CONTRATOS Y SU VALIDEZ LEGAL
}

\section{José A. Vega Sainz}

\author{
Trabajador independiente. Abogado senior y Head of Legal Innovation en Oster Abogados \\ jvegasainz@ius.austral.edu.ar
}

Recibido: 2/04/2020

Aceptado: $2 / 05 / 2020$

\section{Resumen}

De acuerdo con los resultados de un estudio realizado por la IACCM, en la actualidad, los contratos no son redactados ni funcionan como querrían sus usuarios. En general, se pone mucho énfasis en la minimización del riesgo y en los mecanismos de resolución en caso de conflicto, pero no se trabaja a efectos de que el contrato sea una herramienta para que las partes puedan cumplir con sus objetivos comerciales. Dicha situación exige un cambio de paradigma. Una alternativa al paradigma actual es el denominado "derecho preventivo y proactivo", cuyo objetivo es el de prevenir el conflicto antes de que surja, incentivando la colaboración entre las partes e intentando que el contrato sirva como una herramienta a dichos efectos. Para ello, es necesario que los contratos sean verdaderamente comprensibles y utilizables por sus verdaderos usuarios. Una manera de procurar que así sean es mediante la aplicación de herramientas propias del Legal Design Thinking y el diseño contractual, tal como el uso de herramientas visuales: íconos, líneas de tiempo, ciclos, diagramas y tablas, entre otros. Entendemos que la utilización de este tipo de herramientas tiene plena validez legal en tanto que el principio de libertad contractual permite a las partes determinar el contenido, estilo y formato del contrato.

Palabras clave: pensamiento de diseño legal, diseño legal, diseño contractual, derecho preventivo y proactivo, herramientas visuales, visuales, contratos.

\section{Legal Design Thinking, visuals in contracts and its legal validity}

\begin{abstract}
According to the results of recent studies, contracts today are not drafted or work the way its users intend. In general, too much emphasis is made in minimizing risk and not much work is made in order for contracts to serve as tools for the parties to accomplish their commercial
\end{abstract}


objectives. This situation demands a change of paradigm. An alternative to the current paradigm is that of "Proactive and Preventive Law", which objective is to prevent the occurrence of a conflict before it arises, incentivizing collaboration and trying to make contracts serve as a tool for those purposes. In order for this to be accomplished, it is necessary to work to make contracts truly understandable and usable by its true users. One way to procure for contracts to be more understandable and usable is by means of tools that Legal Design Thinking and contract design make available to us, such as visuals tools: icons, timelines, cycles, diagrams and tables, among others. We understand that the use of these kind of tools has full legal validity as the principle of contractual freedom allows for the parties to determine the content, style and format of a contract.

Key words: Legal Design Thinking, legal design, contract design, proactive and preventive law, visual tools, visuals, contracts.

\section{Introducción}

Normalmente, los abogados nos esmeramos en redactar contratos que sean válidos y exigibles, donde se logre minimizar al máximo posible los riesgos de nuestro cliente y en virtud de los cuales este pueda lograr una posición favorable en caso de conflicto. Si bien todos estos fines son loables e importantes, rara vez serán los fines principales de nuestro cliente. Las empresas suscriben contratos con la finalidad de lograr un determinado objetivo comercial o para lograr una colaboración efectiva y beneficiosa con la otra parte. En teoría, no debería existir empresa alguna que suscriba un contrato con la finalidad de litigar y reclamar a la otra parte por daños y perjuicios (Haapio, Plewe y Roy, pp. 2016, 1-2), (Passera, Haapio y Barton, 2013, pp. 2-4) y (Passera, 2017a, pp. 19-20). Entonces, las preguntas surgen por sí mismas: ¿estamos negociando y redactando contratos de forma eficaz? ¿Acaso estamos desviando el foco de atención de nuestros clientes? ¿Deberíamos centrarnos en la minimización del riesgo o más bien en su gestión y la consecución de objetivos comerciales?

Entendemos que los contratos deberían ser negociados y redactados teniendo en mente no solo la posibilidad de conflicto, sino principalmente teniendo en cuenta los objetivos comerciales de las partes. Así, en las negociaciones se debería poner foco en los objetivos que pretenden alcanzar las partes y debería intentarse encontrar oportunidades de sinergia entre ellas. Por otro lado, en los contratos debería plasmarse claramente aquello que se pretende conseguir o lograr, prever los procesos que se llevarán a cabo para alcanzar dichos objetivos y establecer los mecanismos de gestión necesarios para lograr una colaboración efectiva.

Sin embargo, muchas veces, parecería ser que las negociaciones suelen cen- 
trarse en tecnicismos legales, la minimización del riesgo (y no en su gestión), la posibilidad de que existan controversias y la resolución de conflictos. Esto puede generar que la relación entre las partes se recienta ab initio en el proceso de negociación y se pierda la oportunidad para estructurar una relación colaborativa que verdaderamente genere valor. ${ }^{1}$

De acuerdo con los resultados de un estudio realizado por la International Association for Contract \& Commercial Management (IACCM), actualmente, los contratos no son redactados ni funcionan como quisieran sus usuarios. La mayoría de los profesionales que trabajan con contratos comerciales expresan que estos son difíciles de entender y de utilizar (Association for Contract \& Commercial Management [IACCM, 2015).

En dicho estudio, se pone de manifiesto que, al redactar los contratos, normalmente no se enuncian de forma clara los objetivos de las partes y suele faltar un marco en el que se refleje adecuadamente la relación comercial subyacente. Por otro lado, se enumera entre las causas por las cuales los contratos fracasan el hecho de que las negociaciones se tornan innecesariamente extensas y que no se suele involucrar en ellas a todos los partícipes e interesados (stakeholders). También se hace referencia a que el proceso de negociación se centra excesivamente en la mitigación del riesgo, creando un marco de negociación adversarial que no resulta adecuado para fomentar la innovación y creación de valor (IACCM, 2015). ${ }^{2}$

Este marco -tal como es descripto por la IACCM - nos lleva a plantear algunas premisas que pueden servir a efectos de intentar cambiar la situación actual: (a) deberíamos entender que los verdaderos usuarios de los contratos son sus partes y no los abogados y los jueces; (b) el principal marco de actuación de los contratos no son los tribunales, sino el ámbito comercial y empresarial (principalmente en lo que refiere a la gestión de proyectos); (c) el verdadero fin por el que se suscribe un contrato es para que las partes logren conseguir sus objetivos comerciales y generar una verdadera colaboración entre ellas; y

1 Ver Frydlinger, Hart y Vitasek (2019), donde se repasa el camino que transitaron algunas organizaciones para modificar el modelo de contratación que utilizaban, superando relaciones que producían frustraciones y gastos en recursos para pasar a relaciones que producen sinergias y valor agregado para las partes.

2 Asimismo, en Passera (2017a, pp. 102 y ss.), se realizan diversos estudios e investigaciones demostrando que el uso del lenguaje plano y de herramientas visuales hace que los contratos sean más entendibles y mejoren su usabilidad. Los estudios fueron realizados respecto a diversos grupos, tales como estudiantes, empleados públicos y ejecutivos del sector privado, mostrando resultados similares en todos los casos. 
(d) consecuentemente, los contratos deberían ser negociados, diseñados y redactados en vista a lo descripto en los puntos (a), (b) y (c), teniendo en cuenta quiénes son sus verdaderos usuarios, cuál es su marco de actuación y cuáles son las finalidades y objetivos que se pretenden alcanzar.

En el presente trabajo, se introducen los conceptos de Legal Design Thinking (pensamiento de diseño aplicado al Derecho) y el Derecho Proactivo y Preventivo, que entendemos pueden contribuir a dar respuesta a la situación descripta más arriba. Luego, se hace una revisión de distintas herramientas visuales que pueden utilizarse en los contratos para lograr un mejor diseño contractual. Finalmente, se realiza un breve análisis respecto a la viabilidad legal de la incorporación de visualizaciones en los contratos y su posible regulación por las partes.

\section{El derecho proactivo y preventivo y el Legal Design Thinking}

La situación descripta en el apartado anterior exige un cambio de paradigma. Podría decirse que el paradigma actual gira en torno a lo que se suele denominar como "derecho reactivo", que se enfoca en minimizar y mitigar los riesgos asociados a un contrato y a partir del cual se procura negociar y redactar el documento teniendo en cuenta principalmente la posibilidad de que surja un conflicto. A nuestro parecer, el problema de este modelo radica en que hace que sea difícil lograr sinergias y una colaboración efectiva entre las partes.

Una alternativa al paradigma actual es el denominado "derecho proactivo y preventivo", cuyo objetivo es el de prevenir el conflicto antes de que surja, incentivando la colaboración entre las partes e intentando que el contrato sirva como una herramienta a dichos efectos (Passera, 2017a, pp. 21-22). Según este enfoque, los contratos deben actuar como medios que propicien y faciliten los negocios y objetivos comerciales de las partes, que sean instrumentos para facilitar la gestión de proyectos y herramientas de comunicación (Tsygankova, 2016, pp. 1-2), (Passera, 2017a, pp. 19-30) y (Hapio, Plewe y Roy, 2016, pp. 2-3). En líneas generales, la idea es que si los objetivos de las partes son cumplidos, existe una buena colaboración entre ellas y logran implantarse procesos y mecanismos de gestión, es menos probable que surja un conflicto.

Para que estas funciones del contrato puedan ser concretadas, es necesario lograr que los contratos sean realmente comprensibles y utilizables por todos los actores involucrados en su ciclo de vida, incluidos gerentes, jefes de proyecto, financieros y equipos de trabajo, y no solo por los jueces y abogados. Asimismo, si se logra que los contratos sean más claros, fáciles de entender y se mejora su 
usabilidad, es probable que sean más y mejor utilizados por las partes y que existan menos dudas en cuanto a su interpretación (que es una de las principales causas de conflicto). Una manera de lograr estos objetivos puede ser a través de la aplicación de conceptos y herramientas propios del Legal Design Thinking y el diseño contractual. ${ }^{3}$

El Design Thinking -o pensamiento de diseño- es una técnica utilizada por los diseñadores para resolver problemas complejos mediante la comprensión y empatía con el usuario. Es una lente a través de la cual se pueden observar los problemas desde la perspectiva del usuario, para así detectar sus inquietudes y necesidades e intentar darles solución. Dado que se trata de una técnica, es adaptable y puede aplicarse a distintos campos para la resolución de sus problemas: el derecho no escapa a ello. Así, nace una técnica específica, denominada Legal Design Thinking, que pretende aplicar este pensamiento de diseño al campo del derecho, adaptándolo según las problemáticas específicas de este (Hagan, caps. 1-2), (Rossi y Pamirani, 2017, p. 3). Una aplicación del Legal Design Thinking en lo que respecta a la negociación y redacción de contratos es el "diseño contractual".

\section{Diseño contractual y herramientas visuales}

Bajo las premisas del diseño contractual, los contratos no deberían ser solo redactados, sino "diseñados" para que sus verdaderos usuarios (que nosotros entendemos son aquellas personas que intervendrán en la ejecución del contrato, como puede ser un jefe de proyecto o un equipo de trabajo, entre otros) puedan darles un uso real y útil. Esto, además, podría coadyuvar a que los contratos cumplan con su finalidad preventiva de conflictos. ${ }^{4}$

Para poder realizar un efectivo diseño contractual, el Legal Design Thinking pone a nuestra disposición un amplio abanico de herramientas que introduce en el área del diseño contractual, valiéndose de patrones de trabajo propios de los campos del diseño de la información, del diseño de la comunicación y del

3 Cfr. Haapio, Plewe y Roy (2016, pp. 2-3) y Tsygankova (2016, p. 1).

4 Cfr. Passera y Haapio (2013), donde se califica a los contratos como objetos de frontera; Passera, Haapio, Plewe y Roy (2016, p. 3), donde se califica a los contratos como herramientas de comunicación y facilitadores de negocios; Passera (2017a, pp. 21-22, $32-26$ y 50-61), donde, además de referirse a la función preventiva de conflicto de los contratos, se analiza de qué manera la forma en que se negocian, redactan y presentan los contratos puede influir en la percepción de la relación entre las partes, logrando una mayor colaboración y generando sinergias entre ellas; Passera, (2017b), donde también se hace referencia a la función preventiva de los contratos y se los califica como instrumentos para lograr el entendimiento entre las partes, que asisten en la comunicación y colaboración entre las partes y las empodera para lograr sus cometidos. 
diseño de documentos (Passera, 2017a, p. 24), (Passera y Haapio, 2013, p. 38) y (Passera, 2017b, pp. 8-10). Así, por ejemplo, diversas guías y manuales analizan el uso de instrumentos tales como tipografía, tamaño de letra, uso de espacios en blanco, cantidad de renglones por página y técnicas de resaltado, con la finalidad de mejorar la usabilidad de los contratos. ${ }^{5}$

Por otra parte, es posible utilizar herramientas visuales dentro de los contratos, tales como íconos, líneas de tiempo o diagramas, las cuales pueden facilitar la interpretación y lectura de los contratos, especialmente por aquellos usuarios que no poseen conocimientos técnico-legales. ${ }^{6}$ Estos instrumentos deberían permitir que el documento en sí mismo aparezca como más amigable al usuario, invitando a su lectura y facilitando su uso y navegación. ${ }^{?}$

La elección de una u otra herramienta visual en el diseño de un contrato debería estar basada en un análisis previo, donde se defina quiénes serán sus usuarios y qué uso harán del documento. ${ }^{8}$ En este sentido, resultaría aconsejable que se analice el perfil de los usuarios con el fin de indagar cómo utilizarán el contrato y entender a qué dificultades pueden verse expuestos. En base a ello, podrían seleccionarse las herramientas más idóneas para diseñar el contrato adecuadamente.

A continuación, presentamos algunas herramientas visuales que pueden ser utilizadas en el diseño contractual: ${ }^{9}$

- Esquemas de colores: consiste en agregar colores a las distintas cláusulas o secciones de un contrato mediante la incorporación de algún tipo de formato en los títulos y subtítulos, fondos y sombreados, entre otros. El objetivo de la utilización de esta herramienta es lograr un cierto orden en el documento, que asista al usuario a la hora de diferenciar entre las distintas secciones del contrato y facilite su navegación.

- Referencias internas con colores: radica en utilizar colores para identificar

5 Ver Tsygankova (2016), Adams (2013) y Felker et al. (1981), entre otros.

6 Cfr. Passera (2017a), donde el resultado de los estudios realizados demuestra que la utilización de herramientas visuales en los contratos ayuda a: (a) mejorar la comprensión de los contratos; (b) encontrar información relevante de forma más fácil y rápida; y (c) mejorar la usabilidad del documento.

7 Cfr. Passera (2017b), donde se revisa distintos tipos de herramientas visuales y sus aplicaciones.

8 Cfr. Passera y Haapio (2013, pp. 40-41), donde se revisan las distintas dimensiones del diseño de contratos centrados en el usuario y se hace referencia a las necesidades de los usuarios, las características del documento y su contenido.

9 Para ver más sobre la utilización de estas y otras herramientas, ver Passera (2017a, pp. 41-49), donde se pueden encontrar tablas y sus distintos tipos de visualizaciones y propósito; Passera (2017b) y Tsygankova (2016), entre otros. 
las distintas cláusulas o secciones de un contrato y luego, cuando en una cláusula o sección del documento se hace alusión a otra, utilizar el color asignado a esta última. Por ejemplo, si la cláusula segunda del contrato fue identificada con el color verde, al hacer referencia a dicha cláusula más adelante en el contrato, la referencia debería llevar ese mismo color, facilitando así al usuario la navegación del documento.

- Resaltados: es una herramienta a través de la cual se enfatiza determinada cláusula, frase o palabra mediante la utilización de un tipo de fuente distinta, subrayados, cursivas, negritas o colores. Esta herramienta puede utilizarse para dotar de mayor importancia a distintas secciones del contrato o para llamar la atención con respecto a algún término o cuestión específicos.

- Íconos: son imágenes pequeñas y simples, de alto valor representativo, que acompañan a una cláusula escrita del contrato. Los íconos pueden ser utilizados para facilitar la navegación del documento, como así también para lograr una mayor y mejor retención de algún concepto importante. Nunca deben ser utilizados de forma aislada, sino como complemento al texto contractual, ya que no proveen información precisa por sí mismos.

\section{Ejemplo:}

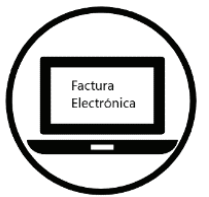

Facturación. El proveedor deberá emitir a favor del cliente una factura electrónica de conformidad con las exigencias legales vigentes al momento de la facturación.

- Líneas de tiempo: consiste en ubicar distintos eventos, plazos o fechas dentro de una línea horizontal que representa un período determinado de tiempo. Normalmente, se utiliza para acompañar una cláusula escrita y tiene como finalidad clarificar el contenido de esta o asistir en su interpretación.

Ejemplo:

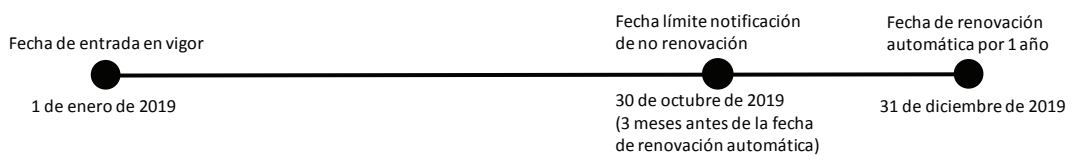


- Ciclos: son una herramienta que se utiliza para graficar una secuencia de eventos que se suceden en el tiempo hasta llegar a un punto final, a partir del cual los eventos vuelven a repetirse en el mismo orden. Los ciclos normalmente son representados en forma circular, a efectos de indicar que, al final, los eventos deben volver a repetirse. De manera similar a las líneas de tiempo, se utilizan para acompañar una cláusula escrita, intentando clarificar su contenido o asistir en la interpretación.

Ejemplo:

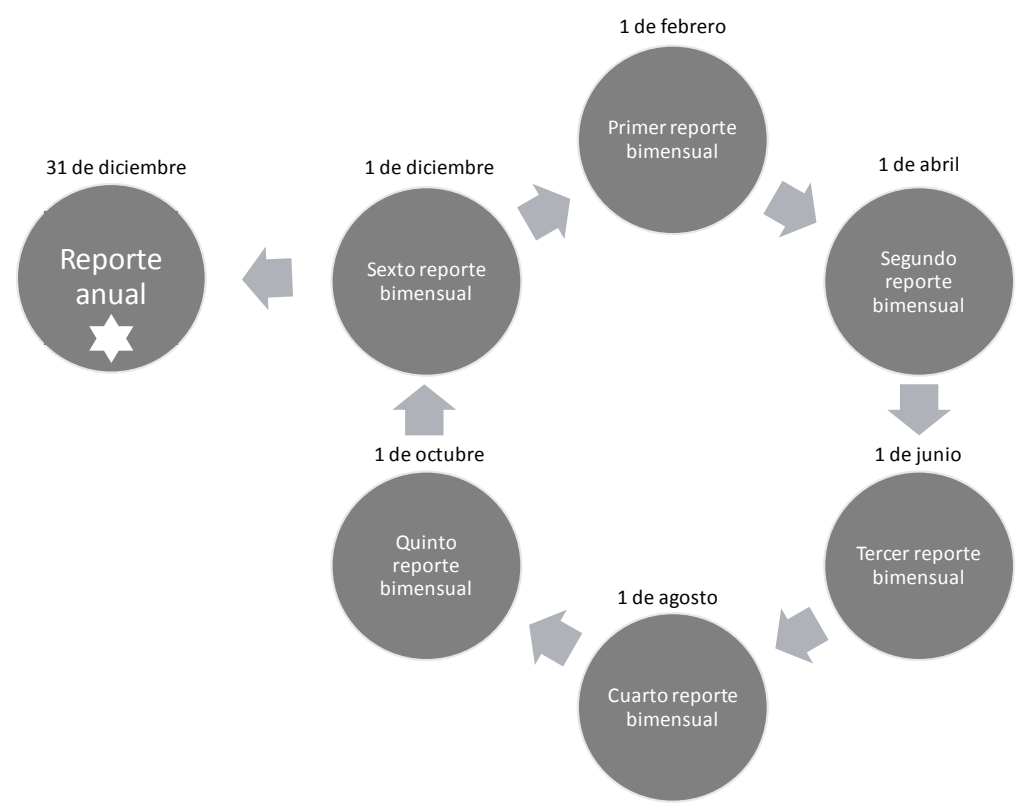

- Diagramas: los diagramas son representaciones gráficas de las distintas variables que pueden ocurrir en una secuencia de eventos o actividades a desarrollar. Resultan sumamente útiles a la hora de representar procesos, ya que muestran de forma gráfica la manera en la que estos se llevarán a cabo y sus distintas variables.

Ejemplo: 


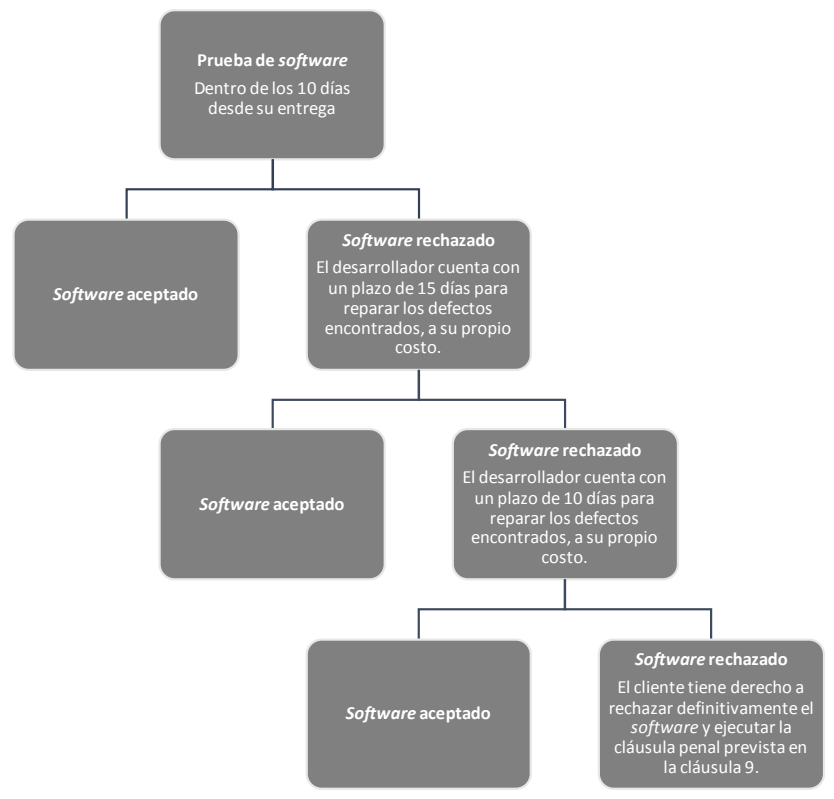

- Tablas: son herramientas que permiten organizar información en líneas y columnas, de modo tal que pueda ser esquematizada o comparada.

Ejemplo:

\begin{tabular}{l|l|l|l}
\hline \multicolumn{1}{c|}{ Quién } & \multicolumn{1}{|c|}{ Derecho de uso } & \multicolumn{1}{c}{$\begin{array}{c}\text { Derecho de } \\
\text { comercializar }\end{array}$} & \multicolumn{1}{c}{$\begin{array}{c}\text { Derecho de crear } \\
\text { obras derivadas }\end{array}$} \\
\hline \multirow{4}{*}{ Licenciatario } & $\begin{array}{l}\text { No tiene derecho a } \\
\text { utilizar el software para } \\
\text { sus actividades propias. } \\
\text { Puede utilizar el } \\
\text { software dentro del } \\
\text { marco de sus activida- } \\
\text { des de comercialización } \\
\text { a clientes finales. }\end{array}$ & $\begin{array}{l}\text { Tiene el derecho de } \\
\text { comercializar el } \\
\text { software a clientes } \\
\text { finales. }\end{array}$ & $\begin{array}{l}\text { No tiene derecho a } \\
\text { realizar obras derivadas } \\
\text { a partir del software ni a } \\
\text { modificarlo. }\end{array}$ \\
\hline Sublicenciatario & $\begin{array}{l}\text { Tiene derecho a utilizar } \\
\text { el software para sus } \\
\text { actividades propias de } \\
\text { acuerdo a lo previsto en } \\
\text { el acuerdo de licencia } \\
\text { modelo incluido en el } \\
\text { Anexo C. }\end{array}$ & $\begin{array}{l}\text { No tiene derecho a } \\
\text { comercializar el } \\
\text { software en ningún } \\
\text { caso. }\end{array}$ & $\begin{array}{l}\text { No tiene derecho a } \\
\text { realizar obras derivadas } \\
\text { a partir del software ni a } \\
\text { modificarlo. }\end{array}$ \\
\hline
\end{tabular}


Otras herramientas visuales que pueden utilizarse son las imágenes e ilustraciones, gráficos, personas y cuadros comparativos, entre otros. Cabe destacar que, en esta materia, rige la libertad de las partes, quienes podrían utilizar cualquier tipo de herramientas visuales que ideen, siempre que estas sirvan adecuadamente a las necesidades de los usuarios del contrato.

\section{El principio de libertad contractual, interpretación e integración de los contratos}

Ninguna norma vigente en la República Argentina regula de manera específica la utilización de herramientas visuales dentro de los contratos. Por esta razón, analizaremos la normativa general contenida en el Código Civil y Comercial de la Nación (CCC) que aplica a los contratos, la formación del consentimiento y la expresión de voluntad de las partes, con el objetivo de intentar discernir si es posible incorporar visuales en los contratos. Asimismo, realizaremos algunas consideraciones sobre las normas contenidas en el CCC respecto a la interpretación e integración de los contratos.

El artículo 957 del CCC define "contrato" como "[...] el acto jurídico mediante el cual dos o más partes manifiestan su consentimiento para crear, regular [...] o extinguir relaciones jurídicas patrimoniales”. Por otra parte, el artículo 958 del mismo cuerpo normativo prevé el principio de libertad de contratación, según el cual "[l]as partes son libres de celebrar un contrato y determinar su contenido [...]”. Finalmente, el principio de la buena fe contractual es receptado en el artículo 961 del CCC, donde se establece que

[l]os contratos deben celebrarse, interpretarse y ejecutarse de buena fe. Obligan no sólo a lo que está formalmente expresado, sino a todas las consecuencias que puedan considerarse comprendidas en ellos, con los alcances en que razonablemente se habría obligado un contratante cuidadoso y previsor.

Conforme a lo previsto en el CCC, el principio de libertad contractual autoriza a las partes de un contrato a determinar libremente el contenido de este, lo que por lógica debe incluir también la posibilidad de determinar su estilo y formato, con la posibilidad de incorporar herramientas visuales en el documento. ${ }^{10}$ Si un contrato es la manifestación de la voluntad de las partes, estas deben tener la libertad de decidir cuál es la mejor manera de manifestar dicha

10 Cfr. Passera (2017a, p. 22), donde se establece que el principio de libertad contractual incluye la posibilidad de las partes de determinar el contenido, estilo y formato del contrato. 
voluntad: si a través de palabras, de visuales o mediante una combinación de ambas. Puede suceder que sea mucho más efectivo e idóneo expresar determinado concepto a través de una herramienta visual, como una línea de tiempo, una imagen o un diagrama, con lo cual debe existir una amplia libertad para que las partes hagan uso de cualquier tipo de herramienta que les permita manifestar su voluntad de la forma más conveniente posible.

Por otro lado, según el principio de buena fe, los contratos obligan a las partes no solo a lo que está formalmente expresado en palabras, sino a todas las consecuencias que podrían considerarse comprendidas en ellas. Una buena manera de poner de manifiesto aquello que no puede expresarse en palabras es a través de visuales. Como hemos dicho, estas herramientas pueden facilitar a las partes la manifestación de su voluntad de forma más clara y precisa en el contrato, permitiendo que se expresen determinadas consecuencias y previsiones que quizás no pueden ser adecuadamente explicitadas de otra manera.

Asimismo, las herramientas visuales también pueden funcionar como un apoyo a la hora de interpretar las palabras. Según se prevé en el artículo 1061 del CCC, "[e]l contrato debe interpretarse conforme a la intención común de las partes y al principio de la buena fe". En este sentido, las visuales que se incorporen a un contrato pueden ser utilizadas a efectos de interpretar el documento conforme a lo que pudo haber sido la intención común de las partes al momento de contratar. Dicha intención puede, en ocasiones, surgir de forma más clara a través de una visual que a través de las palabras.

Asimismo, debemos hacer mención al principio de interpretación contextual contenido en el artículo 1064 del CCC, conforme al cual "[1]as cláusulas del contrato se interpretan las unas por medio de las otras, y atribuyéndoles el sentido apropiado al conjunto del acto". Las cláusulas del contrato no necesariamente deben consistir en texto escrito, sino que las visuales que se incluyan en el documento también pueden ser entendidas como cláusulas en el sentido del artículo 1064 del CCC.

Bajo este entendimiento, no debería leerse el contrato tomando únicamente en consideración su texto escrito, como tampoco debería interpretarse el sentido de las herramientas visuales del contrato de forma aislada. Más bien, se deberá dar un sentido apropiado al conjunto del acto, constituido en este caso tanto por las palabras escritas como por visuales. Cabe destacar que, la mayoría de las veces, las herramientas visuales que se incluyan en un contrato acompañarán al texto escrito de una cláusula o sección con la finalidad de clarificar su contenido o asistir en su interpretación y no existirán de forma independiente. 
Esto hace que deban ser necesariamente interpretadas en conjunto con el texto y no de forma aislada.

Por otro lado, el artículo 1065 del CCC indica que

[c]uando el significado de las palabras interpretado contextualmente no es suficiente, se deben tomar en consideración: a) las circunstancias en que se celebró, incluyendo las negociaciones preliminares; b) la conducta de las partes, incluso la posterior a su celebración; c) la naturaleza y finalidad del contrato.

Las visuales que se incluyan en un contrato servirán como una fuente más de interpretación. Como se explicará más adelante, las visuales pueden surgir a partir de la misma negociación del contrato entre las partes y ser incluidas luego en el documento. Esto podría permitir conocer con mayor exactitud cuál fue la intención de las partes al momento de contratar.

Finalmente, en el artículo 964 del CCC, se entiende que el contenido del contrato se integra con: (a) las normas indisponibles contenidas en el ordenamiento jurídico; (b) la voluntad de las partes; (c) las normas supletorias; y (d) los usos y costumbres del lugar de celebración que sean aplicables, bien porque las partes han decidido incorporarlos al contrato o porque son regularmente observados en el lugar donde se celebra el contrato, en tanto estos no sean irrazonables. Al respecto, diremos:

1. En cuanto a las normas indisponibles, las visuales no podrán estar en contraposición a estas normas. En caso de que así sea, deberán tenerse como no incluidas dentro del contrato.

2. En relación con la voluntad de las partes, ya hemos dicho que las herramientas visuales son un medio más a través de las cuales puede expresarse dicha voluntad. Por lo tanto, debe entenderse que las visuales integran el contrato.

3. Las normas supletorias solo deberán integrar el contrato cuando una determinada cuestión no se encuentre regulada por la voluntad de las partes, sea esta expresada por medio de palabras o de visuales.

4. Las visuales pueden ser una gran herramienta para referenciar o incorporar usos y costumbres al contrato. Quizás, el ejemplo más claro de esto son los incoterms, respecto a los cuales existe una gran cantidad de infografías donde se explica cómo funciona cada uno. Estas infografías podrían ser incorporadas al contrato o a sus anexos en caso de que las partes lo estimen conveniente. ${ }^{11}$

11 Ver Thompson (2020), entre otros. 


\section{Regulación de las herramientas visuales en los contratos}

Conforme lo hemos expuesto, la inclusión de herramientas visuales dentro de los contratos debe ser considerada válida. Entendemos que el principio de libertad contractual permite a las partes expresar su voluntad de la manera que estimen más adecuada, tanto en palabras como a través de otros medios. Asimismo, opinamos que las visuales deben integrar el contrato y que al momento de interpretar el documento debe tomarse en consideración tanto el texto escrito como las herramientas visuales. Sin perjuicio de lo anterior, resulta posible que, en determinadas ocasiones, las visuales contradigan el texto contractual. De esta manera, resultaría conveniente regular la inclusión de estas herramientas dentro del contrato.

Actualmente, en la práctica contractual suelen agregarse títulos a las cláusulas con el propósito de facilitar la lectura y navegación del documento. Asimismo, es usual regular el uso que se hace de esta herramienta bajo una cláusula que normalmente se denomina "títulos" o "encabezados", donde se establece que "los encabezados incluidos en el contrato han sido incluidos únicamente por razones de conveniencia y no tienen efecto sustancial en la interpretación del contrato”. Por otra parte, en caso de que el contrato sea redactado en dos idiomas, suele establecerse una cláusula donde se le otorga prevalencia a una u otra versión, del siguiente modo: "el contrato se realiza tanto en idioma español como en inglés. Sin embargo, la versión en español prevalecerá en el caso de un conflicto en el que surjan discrepancias o inconsistencias entre los términos de ambas versiones".

Si tomamos como base las técnicas de regulación a las que hemos hecho referencia en relación con los encabezados e idiomas, podemos ensayar un modelo de cláusula que rija el uso de herramientas visuales en el contrato. ${ }^{12} \mathrm{~A}$ continuación, presentamos nuestra propuesta de cláusula modelo, sin perjuicio de que las partes de un contrato podrían adoptar alguna otra postura según las particularidades de cada caso en concreto:

Las herramientas visuales incluidas en el presente contrato (tales como el uso de colores, tipografías, diagramas, tablas e íconos, entre otros) son válidas y expresan la voluntad de las partes. En consecuencia, estas integran el contrato y surtirán efectos para su interpretación junto con el texto escrito del acuerdo. Sin perjuicio de lo anterior, en caso de que surja un conflicto basado en

12 Cfr. Passera y Haapio (2013, p. 44), donde se prevé que, en caso de conflicto, podría prevalecer el texto contractual o las visuales, tal como sucede en los casos en los que el contrato tiene versiones en varios idiomas y se establece que una versión debe prevalecer sobre las otras. 
discrepancias o inconsistencias entre el texto escrito del contrato y las herramientas visuales incluidas en este, deberá prevalecer [lo establecido en el texto escrito del contrato/lo previsto en las herramientas visuales incluidas en el contrato].

\section{Aplicación práctica}

El proceso de diseño contractual tendría que empezar desde el momento de la negociación del contrato, donde debería ponerse de manifiesto cuál es la intención de las partes para contratar y cuáles son sus objetivos, para que así estos puedan ser alcanzados, implementando procesos y mecanismos de gestión y procurando que se generen sinergias entre las partes. Asimismo, sería conveniente involucrar en el proceso de negociación a todos los posibles usuarios del contrato, procurar conocerlos e indagar sobre sus necesidades y requerimientos. La finalidad sería diseñar un contrato que efectivamente logre satisfacer sus necesidades.

En el proceso de negociación contractual, también pueden utilizarse herramientas de Legal Design Thinking a efectos de encaminar las negociaciones, poner de manifiesto los objetivos, necesidades y preocupaciones de las partes, fomentar la colaboración, aclarar el entendimiento que cada una de ellas tiene con respecto a alguna cuestión específica, etc. Esto, además, tiene una ventaja inconmensurable, que radica en el hecho de que si las partes utilizan visuales en la negociación, estas pueden ser guardadas para luego ser incorporadas en el contrato.

Por ejemplo, si las partes en una reunión de negociación se encuentran discutiendo sobre cómo implementar determinado proceso y nosotros, como abogados, recomendamos la utilización de un diagrama para graficar ese proceso y trabajarlo en la reunión, podremos luego incorporarlo como parte del futuro contrato, en donde podría quedar plasmada de forma mucho más clara la intención de las partes. ${ }^{13}$

Luego, una vez que se tenga claro cuáles son los objetivos y las necesidades de las partes, se conozca a todos los partícipes e interesados y haya quedado de manifiesto cuál es el entendimiento que hay con respecto a los distintos preceptos que se incluirán en el contrato, se podría avanzar con el efectivo diseño del contrato. Sería de buena práctica iniciar esta etapa con una sesión de lluvia de ideas (brainstorming) (si es posible, con un grupo interdisciplinario) a efectos de determinar cuáles son las herramientas -visuales o no- más adecuadas para

13 Cfr. Passera (2017a, pp. 142-146). 
utilizar en el diseño del documento. Luego de ello, y de acuerdo al resultado de esa reunión, podríamos avanzar con la redacción del contrato.

En nuestra opinión, deberíamos apuntar a elaborar un contrato flexible, donde se establezca claramente cuáles son los objetivos de las partes, los mecanismos y procesos que se utilizarán para la consecución de dichos objetivos y las formas de gestión que habrá en caso de conflicto. En definitiva, deberíamos procurar elaborar un contrato que sirva como una herramienta para fomentar la colaboración entre las partes y facilitar la concreción de sus metas, intentando disminuir la posibilidad de que surja un conflicto.

Sin perjuicio de lo anterior, la eventualidad del conflicto siempre existirá. En consecuencia, más allá de que se utilicen herramientas visuales en el contrato o un lenguaje más plano, se debería siempre mantener el rigor técnico necesario para asegurar la plena validez de todos los términos incluidos en el documento. En caso de que surjan dudas en el proceso judicial (o bien de mediación o arbitral) respecto al sentido o funcionamiento de las herramientas visuales incorporadas en un contrato, se podría citar a las partes o bien a un experto en diseño de la información con el fin de aclarar la cuestión. En definitiva, las visuales deben ser siempre un elemento más para interpretar el contrato e intentar desentrañar cuál fue la verdadera intención de las partes al momento de contratar y no una cuestión que genere mayor complejidad en esta labor.

\section{Reflexiones finales}

El Legal Design Thinking provee al jurista de una nueva técnica y una enorme cantidad de herramientas que puede utilizar para realizar su labor con mayor eficiencia. Sin embargo, para aprovechar estas nuevas herramientas, debemos estar dispuestos a instruirnos en esta materia, dejar de lado nuestros prejuicios y cambiar nuestra manera de trabajar. Por otra parte, no desconocemos que la adopción de estas nuevas herramientas requerirá del análisis académico, doctrinario y jurisprudencial, tanto para dar luz sobre la mejor manera de utilizarlas como para dar certeza sobre su viabilidad legal. ${ }^{14}$

El presente trabajo no pretende ser sino una primera aproximación al tema, por medio de la cual se intenta introducir los conceptos del derecho proactivo y preventivo, el Legal Design Thinking y el diseño contractual, sobre los que poco

14 Por ejemplo, se ha pregonado la posibilidad de que existan contratos meramente visuales en determinados campos para asistir a grupos de analfabetos o con dificultades de entendimiento. 
se ha escrito en el ámbito del derecho argentino. ${ }^{15}$ La esperanza es que, en el futuro, surjan más investigaciones sobre estos temas y se abra el debate sobre su uso e implementación en la práctica.

\section{Bibliografía}

Adams, K. (2013). A Manual Style for Contract Drafting. Estados Unidos: American Bar Association.

Dore, T. G. (2020). Legal Design Thinking. Un nuevo enfoque para el proceso de familia. Suplemento especial. Derecho, Innovación y Tecnología, 111-120.

Felker, D., Pickering, F., Charrow, V. y Holland, M. (1981). Guidelines for Document Designers. Washington, D. C: American Institutes for Research.

Frydlinger, D., Hart, O. y Vitasek, K. (2019). A New Approach to Contracts. Harvard Business Review. https://hbr.org/2019/09/a-new-approach-to-contracts.

Haapio, H., Plewe, D., y Roy, R. (2016). Next Generation Deal Design: Comics and Visual Platforms for Contracting. En Schweighofer, E. et al. (Eds.), Networks. Proceedings of the $19^{\text {th }}$ International Legal Informatics Symposium IRIS 2016. Viena, Austria, 373-380.

Hagan M., Law by Design. http://www.lawbydesign.co/en/home.

International Association for Contract \& Commercial Management. (IACCM) (2015). Ten Pitfalls to Avoid in Contracting. http://cdn2.hubspot.net/hubfs/483419/Free_Resources/J11652-10Pitfalls-16pp-2015-06-08-web.pdf?__hstc=149696361.d81122da2fac7b3a070fd5a0a0c4f755.1583318203608.1583318203608.1583318203608.1\&_hssc $=149696361.1 .1$ $585754871265 \&$ \&_hsfp=3273370589\& \&hCtaTracking $=6$ b863b06-7c44-486b-9558-e3a2dceee0ea\%7Cde6bf51f-acf3-47cb-aaae-d6620dadd564.

Passera, S. (2017a). Beyond the Wall of Contract Text. (Disertación doctoral). Aalto Publication Series.

Passera, S. (2017b). Contracts as Interfaces: Exploring visual representation patterns in contract design. Cambridge: Cambridge University Press.

Passera, S. y Haapio, H. (2013). Transforming Contracts from Legal Rules to User-Centered Communication Tools: a Human-Information Interaction Challenge. Communication Design Quarterly. https://doi.org/10.1145/2466489.2466498.

Passera, S., Haapio, H. y Barton, T. D. (2013). Innovating Contract Practices: Merging Contract Design with Information Design. Proceedings of the 2013 Academic Forum on Integrating Law and Contract Management: Proactive, Preventive and Strategic Approaches.

Rossi, A. y Palmirani, M. (2017). From Words to Images Through Legal Visualization. Lecture Notes in Computer Science, 10791, 72-85.

Thompson, B. (2020). Incoterms® 2020 Explained - The Complete Guide. https://incodocs. $\mathrm{com} / \mathrm{blog} /$ incoterms-2020-explained-the-complete-guide.

Tsygankova, T. (2016). Design of Good Commercial Contracts - Practical Tools for Contract Drafters. Schweighofer, E. et al. (Eds.), Networks. Proceedings of the $19^{\text {th }}$ International Legal Informatics Symposium IRIS 2016. Viena, Austria, 407-414.

15 De nuestra investigación surge que el único trabajo que se ha publicado en la materia respecto al Legal Design Thinking es Dore (2020, pp. 111 y ss.). 